\title{
Three-phase diode rectifier with current modulator in DC circuit based on multi-channel converter
}

\author{
Michal KRYSTKOWiak, Michal GWóźdź \\ Poznan University of Technology \\ e-mail: Michal.Krystkowiak@put.poznan.pl
}

(Received: 16.11.2016, revised: 08.03.2017)

\begin{abstract}
In the paper the 3-phase power diode rectifier system with quasi-sinusoidal input (power grid) current is presented. In order to benefit from it, the current modulation in the DC circuit of a rectifier is used. The essential part of the current modulator is the wide-band power electronics controlled current source based on a multi-channel converter. The control algorithm of the current modulator respects impact of aliasing phenomena at system stability by using the method proposed by the authors. The paper includes the rectifier system description and rules of operation of the current modulator. Also, some results of research on both the rectifier simulation model and the laboratory prototype of a current modulator are presented.
\end{abstract}

Key words: control methods of electrical systems, diode rectifier, generalized sampling expansion, multi-channel converter, PWM

\section{Introduction}

The power rectifiers belong to a very widely utilized group of power electronics converters. Unfortunately, a standard diode and thyristor rectifier operation is the reason for distortion of currents and voltages in a power grid. It has caused a very serious problem for the energetic system for many years thus, improving quality of input (power) grid current of a rectifier is often necessary. One of the solutions to obtain it is to apply a rectifier based on a passive or active filter at the input [1-5]. The following way relies on the use of an active rectifier, which is built with a transistor inverter switching at frequency, being several times higher than frequency of voltage in a power grid [3,6]. Other proposed solutions use the PFCs based on multi-level converters [7,8]. Also, to correct the waveform of the power grid current the rectifier built with diodes (or thyristors) being interconnected by means of coupled inductors can be used [9]. Another solution of the rectifier system includes the voltage modulator in the DC circuit of a rectifier. The modulator is built with thyristors and a special inductor with several taps. This has already been considered before, e.g. in $[10,11]$. 
All the mentioned solutions are relatively expensive ones. In the paper another possible solution, with the ability to shape the waveform of a power grid current towards the sinusoidal waveform is presented (Fig. 1). This one is based on the current modulation in the DC circuit $[7,8]$. The rules of such system's operation are described in details in e.g. [11]. Therefore, only a brief description of this one is given here for reader's convenience.

The system is built with two 6-pulse diode rectifiers, which are supplied by two 3-phase transformers with "star-star" and "star-delta" connections. In this way 30 el. deg. phase shift of transformers output voltages is obtained. Additionally, in the DC circuit the special power electronics converter called the "current modulator" is placed. The modulator is responsible for shaping rectifier input currents. In fact the "star-star" connected transformer is not necessary for proper operation of the modulator although it helps to scale the voltage levels of both rectifiers in the laboratory prototype of the system [10].

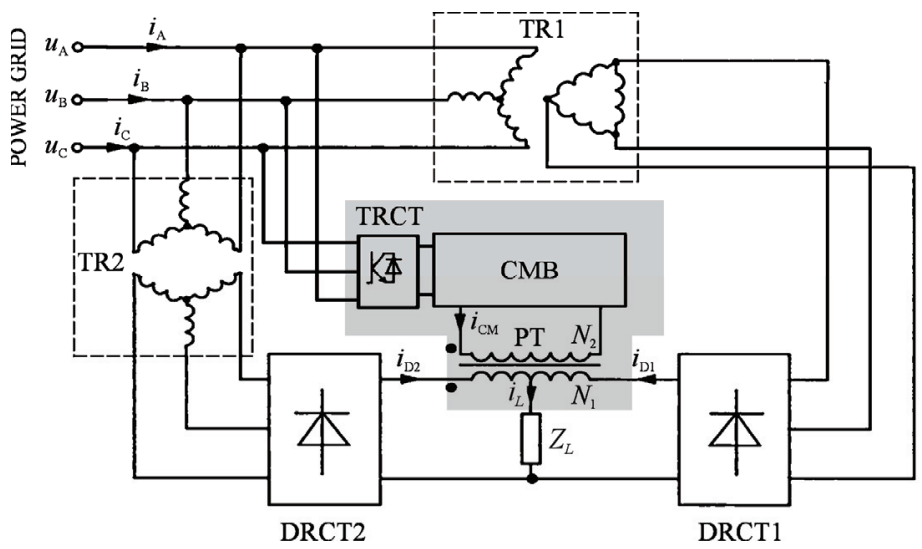

Fig. 1. General block diagram of 3-phase diode rectifier with current modulator in DC circuit

The rectifier system consists of the following blocks: two transformers connected in the star-delta (TR1) and star-star (TR2) manner, two standard 6-pulse diode rectifiers (DRCT1 and DRCT2) with DC circuits connected in parallel, the active (transistor) rectifier (TRCT) and the current modulator block (CMB). The CMB is connected to the DC circuit across the pulse transformer (PT). Rectifiers power a common load, expressed by $Z_{L}$. Similarly like the TR2 transformer, the transistor rectifier is not necessary in the rectifier circuitry too. Its role depends on control of the voltage level in the DC link of the modulator only - without any impact on the modulator output current [10].

The presented solution of the rectifier system makes it possible to improve the quality of rectifier power grid currents $i_{A}, i_{B}$ and $i_{C}$. That is to say, waveforms of these are close to the sinusoidal shape. The current modulator works as a power electronics controlled current source being connected to the DC circuit of rectifiers through a wide-band pulse transformer with two taps on a primary site. The $i_{C M}$ output current of the modulator is added (with a sign of "plus" or "minus"), with the aid of the PT, to the output current $i_{D 1}$ and $i_{D 2}$ of each rectifier in terms of the following equations: 


$$
\left(i_{D 2}-i_{D 1}\right) N_{1}=i_{C M} N_{2}, \quad i_{D 1}=\frac{1}{2}\left(i_{L}-\frac{N_{2}}{N_{1}} i_{C M}\right), \quad i_{D 2}=\frac{1}{2}\left(i_{L}+\frac{N_{2}}{N_{1}} i_{C M}\right),
$$

where: $N_{1} / N_{2}$ - the PT windings turn ratio, $i_{L}$ - the load current and $i_{C M}$ - the $C M B$ output current.

This is the way in which waveforms of input currents of two rectifiers are modified. In consequence, resultant waveforms of power grid currents of the rectifier system $\left(i_{A}, i_{B}\right.$, and $\left.i_{C}\right)$ are modified as well. The power of each, the active rectifier and the current modulator is only $2 \div 3 \%$ of the total power at the output of the DC circuit. This is a great advantage of this idea of the rectifier system.

In order to obtain the sinusoidal shape of the power grid current, the modulator current $i_{C M}$ should satisfy the following equation [11]:

$$
i_{C M}(t)=I_{L} \frac{\frac{6}{\pi} \cos \left(\omega_{g} t\right)-\sum_{k}\left[\cos \left(6 k \omega_{g} t\right)+\cos \left(6 k \omega_{g} t+\frac{\pi}{6}\right)\right]}{\sum_{k}\left[-\cos \left(6 k \omega_{g} t\right)+\cos \left(6 k \omega_{g} t+\frac{\pi}{6}\right)\right]} \frac{N_{1}}{N_{2}} .
$$

However, the complex current form of (2) can be replaced by a current with triangular shape with only a small deterioration of the THD factor of a rectifier input current [11]. The fundamental frequency of the modulator current has to be equal to 6-times of power grid frequency, i.e. $300 \mathrm{~Hz}$ :

$$
i_{C M}(t)=\frac{4}{\pi} I_{L}\left[\sin \left(6 \omega_{g} t\right)-\frac{\sin \left(36 \omega_{g} t\right)}{3^{2}}+\frac{\sin \left(56 \omega_{g} t\right)}{5^{2}}-\ldots\right],
$$

where $\omega_{g}$ is the fundamental frequency of the voltage in the power grid.

With such replacement, assuming the power grid is a symmetrical one, a THD factor of a power grid current is equal to about $1 \%$ [11]. Thus, the current modulator has to include in its own structure a wide-band power electronics controlled current source which would be able to match precisely an output (modulator) current in the reference signal. This feature of the modulator is essential for proper working of the rectifier system because it determines directly the quality of its input current.

Initially, in the current modulator the standard (i.e. one-channel) inverter has been implemented. This one has been a part of the laboratory prototype of rectifier system [10]. The presented article is focused mainly on a wide-band power electronics controlled current source based on a multi-channel (interleaved) converter, being utilized in a current modulator.

The whole text is divided into 6 sections. The first one deals with a general conception of rectifier construction. The second one shows a basic description of the wide-band power electronics controlled current source based on a multi-channel converter. In the third section, an issue of controlled source stability is considered. The fourth section presents mainly, among other items, a simulation model of the rectifier system. In the fifth section selected results of 
research on a laboratory prototype of the current modulator are presented. The last part is dedicated to conclusions.

\section{Wide-band power electronics controlled current source}

Dynamic changes of parameters of both energy source and load are the reasons for decreasing exactitude of output signals towards reference signals. In order to improve these parameters more advanced solutions of power electronics converters are often necessary. They can be exemplified by a wide-band power electronics voltage-controlled voltage source (VCVS) or voltage-controlled current source (VCCS). Such a converter should match an output signal precisely in a reference waveform so that both a modified electrical structure of a converter and an effective control algorithm are necessary. It has many applications in power electronics equipment.

In Fig. 2 a general structure of a VCCS is shown. It is based on the conception of a multichannel converter where a $i_{L}$ total output current is proportional to the sum of currents $i_{L, i}$ : $i=0,1, \ldots, M-1$ in individual channels of a converter.

The VCCS is a system which works in a closed, current type, negative feedback loop. Inverters in an execution block of the VCCS are controlled in PWM mode with the constant value of carrier frequency $[3,12,13]$. One of the fundamental blocks of the VCCS is a passive low-pass filter at the output of power stage consisting of a set of connected in parallel inductors. This filter has two basic tasks to do, namely it obtains the suitable value of the output impedance of a converter and minimizes magnitude of PWM carrier components in the output current, making it possible for the converter to meet requirements of EMC.

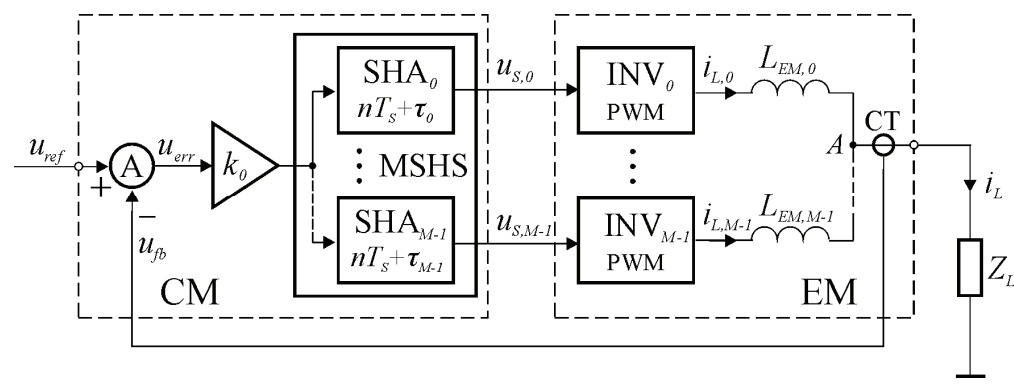

Fig. 2. Block diagram of VCCS

The general structure of the VCCS is based on two modules, the control module $(C M)$ and the execution module $(E M)$. The control module includes the following internal blocks:

- adder $(A)$, producing the error signal $u_{\mathrm{err}}=u_{\mathrm{ref}}-u_{\mathrm{fb}}$,

- regulator of the output current with the gain factor of $k_{0}$,

- $M$-order multi-dimensional sample-and-hold system (MSHS) consisting of $M$ connected in parallel sample-and-hold amplifiers.

The execution module consists of: 
- $M$-connected in parallel half-bridge type inverters,

- output filter $\left(L_{E M, i}: i=0.1, \ldots, M-1\right)$,

- current transducer $(\mathrm{CT})$, producing the feedback voltage $u_{\mathrm{fb}}$, being proportional to the VCCS output current $i_{L}$.

The $E M$ is loaded by the $Z_{L}$ impedance and $Z_{L}=j \omega L_{L}+R_{L}$. The VCCS is controlled by the reference voltage $u_{\text {ref }}$ at the input of the $C M$.

The sampling moments and PWM carrier signals in individual channels of the converter are shifted with each other by $T_{s} / M$, where $T_{s}$ is a master sampling period. As a result, from the point of view of system stability, the converter "transfer function" is preferably modified [12]. Thanks to this, the regulator gain in the VCCS control system can be increased compared to the one-channel converter. It has a very positive impact on the performance of the VCCS because a control algorithm allows for more accurate match of the VCCS output current in a reference one.

In this particular case of operation of the VCCS in a rectifier system its structure has been slightly modified. It has been necessary due to the way of including the pulse transformer in this system based on a virtual ground. As a result, the $2 \times 2$-channel modified inverter in the $E M$ of the VCCS has been used. The block scheme of the VCCS is presented in Fig. 3.

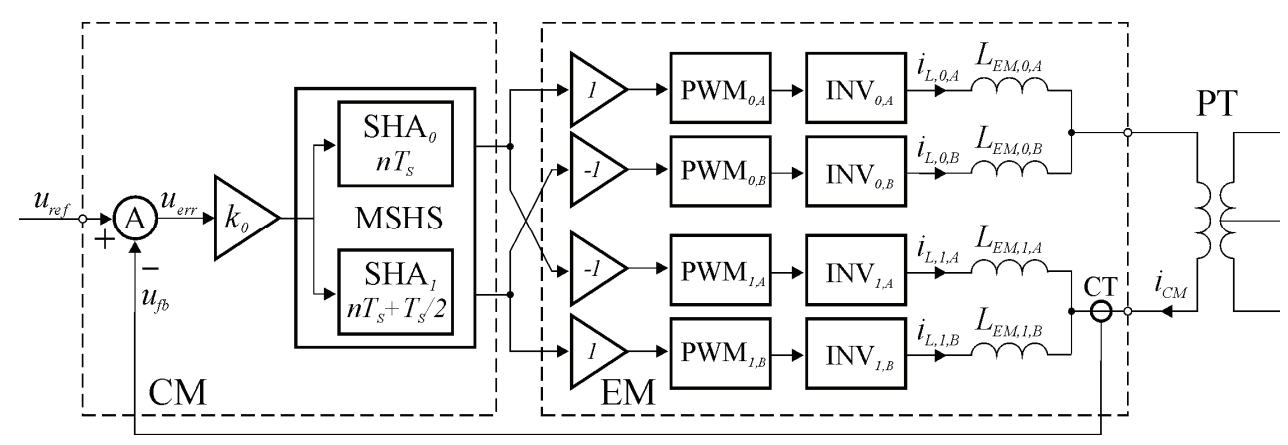

Fig. 3. Block diagram of VCCS for driving pulse transformer

The modulation used in the pulse modulators is two-sided and asymmetric. The load of the VCCS - the pulse transformer - is included in the circuit in a differential manner. That is to say, the voltage at the output of the $\mathrm{INV}_{0, x}$ inverters pair has to be in anti-phase with respect to the voltage at the output of the $\mathrm{INV}_{1, x}$ pair. Because of $M=2$, sampling moments in the $\mathrm{SHA}_{1}$ and $\mathrm{SHA}_{0}$ should be shifted with each other by a $T_{s} / 2$ time.

\section{Stability issue of a controlled current source}

One of the most important aspects of the current modulator work is its stability. This analysis will include an important factor that occurs in the operation of a real system, which usually is not respected. This is an aliasing phenomenon - characteristic for sampled-data systems. The mathematical model of a control system of a multi-channel converter for the stability 
analysis has been proposed e.g. in [12]. This description is based on the extension of the Whittaker-Kotelnikov-Shannon (WKS) sampling theory - the Generalized Sampling Expansion (GSE) - being formulated by Papoulis [14].

Assume signal $x(t) \in L^{2}(\Re)$, the space of the square-integrable functions, where $\Re$ is a real number domain, and its Fourier transform $X(j \omega)$ exists. Assume also that sampling of $x(t)$ is uniform and ideal (i.e. with utilization of Dirac series:

$$
\sum_{n} \delta\left(t-n T_{s}\right): T_{s} \leq \frac{\pi}{\omega_{\max }},
$$

where $T_{s}$ is the sampling period, and $y(t)$ is the signal at output of a sample-and-hold amplifier (SHA) as a 0 -order extrapolator of a sampled signal. The relationship between $X(j \omega)$ and $Y(j \omega)$ apart a static (time invariant) component, also contains a dynamic (time variant) component, i.e.

$$
\sum_{n=-\infty}^{\infty} X\left[j\left(\omega-n \frac{2 \pi}{T_{S}}\right)\right],
$$

so the formal transfer function of the SHA does not exist. The dynamic component is also related to the aliasing effects, by which the high frequency poles are folded back into lower frequencies. Although respecting the static part only of equivalent transfer function of SHA gives, in most cases of a system stability analysis, satisfying results, the crucial knowledge is that the aliasing mechanism can cause loss of stabilization at critical frequencies $[12,15,16]$. From the point of view of the GSE, general setting is that a signal $x(t)$ is processed by a linear multi-dimensional sampling system (MSS). Suppose now that $x(t)$ is a common input to $\mathrm{M}$ sampling systems and each individual sampling system is a sub-system of the MSS - Fig. 4.

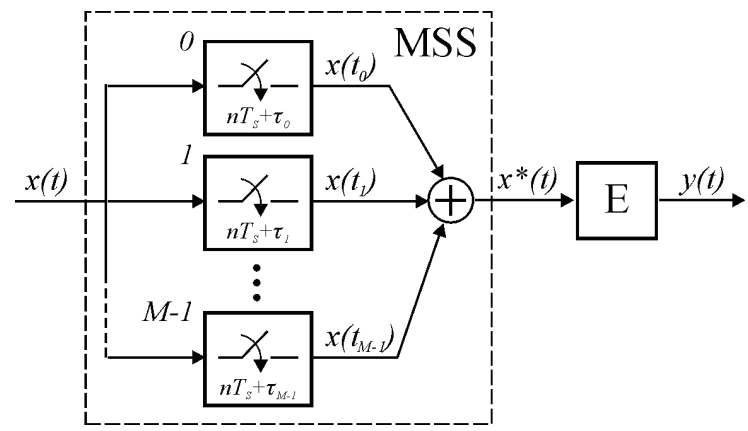

Fig. 4. MSS with single ZOH block as signal extrapolator (E)

Each sub-system samples at a rate of $1 / M$ times of the Nyquist rate. Assuming individual delay

$$
\tau_{i}=i \frac{T_{s}}{M}: i=0,1, \ldots, M-1,
$$

the $x(t)$ can be retrieved from its samples $x^{*}(t)$ by using the inverse Fourier transform formula: 


$$
x(t)=\frac{1}{2 \omega_{\max }} \sum_{n=-\infty}^{\infty} \sum_{i=0}^{M-1}\left\{x\left[\left(n+\frac{i}{M}\right) T_{s}\right]_{-\omega_{\max }}^{\omega_{\max }} e^{j \omega\left[t-\left(n+\frac{i}{M}\right) T_{s}\right]} \mathrm{d} \omega\right\} .
$$

The overall sampling rate still satisfies the Nyquist criterion [14]. The MSS, being the consequence of GSE, makes it possible to reduce the required sampling frequency to $1 / M$, comparing to one-dimensional sampling system (WKS sampling theorem), working at the same sampling rate. Considering the system from the other side, when sampling it at a rate of $1 / T_{s}$ its effective sampling rate is equal to $M / T_{s}$. A Nyquist band is now $M$-times extended. In the case of a control system of a real multi-channel converter an essential difference in relation to GSE assumption appears. It consists in the fact that each individual converter channel includes a single SHA and individual channels output signals are summed at the output of the converter. This modification of the original GSE concept is necessary due to the different position in the system of a signals summing node. The second reason results from the limitation of dynamic parameters of real power electronics devices used in inverters. Thus, the VCCS control system includes now the multidimensional sample-and-hold system (MSHS) instead of the MSS [13]. The proposed small-signal (linear) model of the VCCS with the $2^{\text {nd }}$ order MSHS, being the object of further considerations, is shown in Fig. 5.

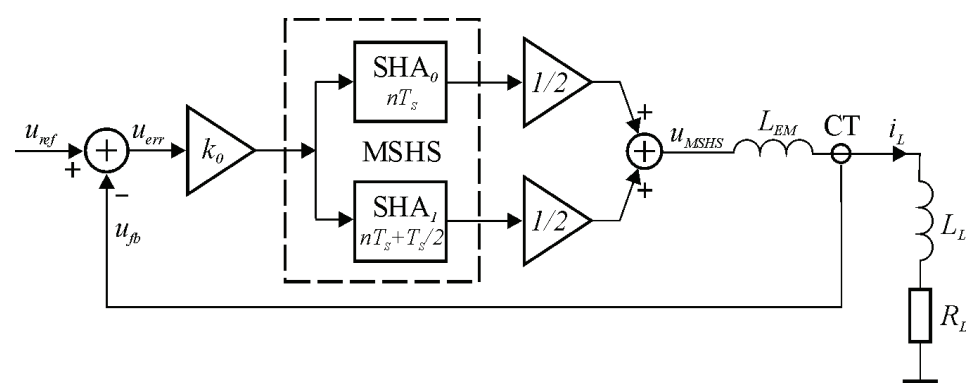

Fig. 5. Small-signal model of VCCS based on 2-channel converter

The proposed model is an IOM, SISO and LTI one and can be completely described in terms of a continuous-time system with a transfer function

$$
G(j \omega)=\frac{I_{L}(j \omega)}{U_{\mathrm{ref}}(j \omega)}=\frac{K(j \omega)}{1+K_{a l}(j \omega)} .
$$

The system stability analysis takes advantage of the Nyquist criterion, e.g. [17]. Hence, the characteristic equation $R(j \omega)=1+K_{a l}(j \omega)=0$ of the model, respecting (4), takes the form:

$$
R(j \omega)=1+r_{C T} k_{0} \sum_{n=-N_{\max }}^{N_{\max }} \frac{K_{\mathrm{MHS}}\left[j\left(\omega-n \omega_{s}\right)\right]}{j\left(\omega-n \omega_{s}\right)\left(L_{E M}+L_{L}\right)+R_{L}}=0,
$$


where: $K_{\mathrm{MHS}}(j \omega)$ is the static component in a relationship of the MSHS input and output signals, $r_{C T}$ is the gain factor of $C T, k_{0}$ is the gain of the regulator, $\omega_{s}$ is the sampling frequency and $N_{\max }$ is the number of respected aliases.

Research of the VCCS small-signal model have defined the static component in the relationship of input and output signals of the MSHS as follows:

$$
K_{\mathrm{MHS}}(j \omega)=\left\{\begin{array}{l}
\mathrm{Sa}\left(\omega \frac{T_{S}}{2}\right) \mathrm{e}^{-j \omega \frac{T_{s}}{2}}: M=1 \\
\mathrm{Sa}\left(\omega \frac{T_{s}}{2}\right) \cos \left(\omega a T_{S}\right) \mathrm{e}^{-j \omega \frac{T_{s}}{2}}: M=2, a=\frac{1}{2} \frac{\tau_{0}-\tau_{1}}{T_{s}} \wedge a \in\left\langle-\frac{1}{4}, \frac{1}{4}\right\rangle
\end{array} .\right.
$$

It is crucial for further utilization of a multi-channel inverter concept to determine the maximal regulator gain $\left(k_{0}\right)$ to make the system stable. In order to determine this value it is necessary to determine magnitude of $K_{a l}(j \omega)$ for the critical phase value $\arg \left\{K_{a l}(j \omega)\right\}= \pm \pi$. It occurs at a frequency of

$$
\omega_{\pi, n}=(2 n+1) \frac{\pi}{T_{s}}: n=0, \pm 1, \pm 2, \ldots .
$$

Having solved (5) the maximal regulator gain can be expressed by the following formula:

$$
k_{0, \max }<\left\{\begin{array}{l}
\frac{L_{E M}+L_{L}}{r_{C T} T_{S}}: M=1, M=2 \wedge a=0 \\
2 \frac{L_{E M}+L_{L}}{r_{C T} T_{S}}: M=2 \wedge a= \pm 1 / 4
\end{array} .\right.
$$

The maximal theoretical value of the regulator gain is 2-times over the gain of the regulator in the case of a one-channel converter. It gives higher quality mapping of the VCCS output current in the reference signal.

\section{Simulation model of rectifier system}

The fundamental block of a current modulator is the VCCS based on the 2-channel converter that powers a pulse transformer [10]. Selected waveforms in the simulation model for the target shape (i.e. triangular) of the reference signal are shown in the following figure are shown in Fig. 6. These are also related to the two cases of an order of the MSHS i.e. $M=1$ and $M=2$. The PWM carrier frequency $T_{c}=100 \mu \mathrm{s}$. The nominal magnitude value of the reference voltage $A_{\text {ref }, n}=10 \mathrm{~A}$, and $L_{E M, 0, A}=L_{E M, 0, B}=L_{E M, 1, A}=L_{E M, 1, B}=1.2 \mathrm{mH}$. Parameters of the simulation model are consistent with parameters of the real system, being described in Section 5 . 
a)

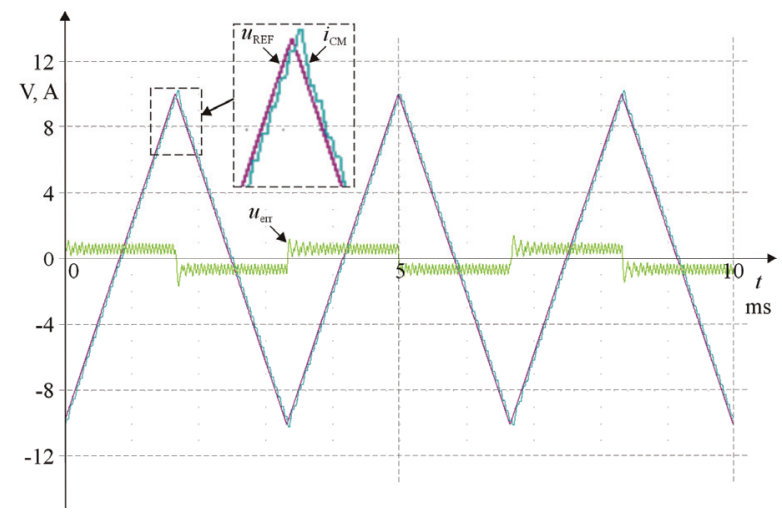

b)

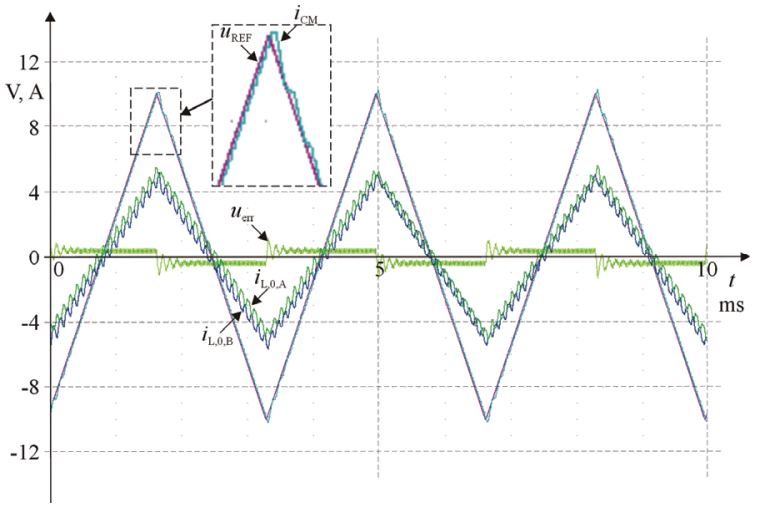

Fig. 6. Waveforms of reference voltage $u_{\text {ref }}$, modulator current $i_{C M}$, and error signal $u_{\text {err }}$ for the case of triangular shape of a reference signal and: a) $M=1, k_{0}=35$; b) $M=2, a=1 / 4 ; k_{0}=40$. Also currents in individual channels of VCCS $\left(i_{L, 0, A}\right.$ and $\left.i_{L, 0, B}\right)$ are shown in Fig. b). Magnitude of the reference signal is equal to nominal one

In the case of the two-channel VCCS shapes of the modulator current and reference signal almost coincide, in contrast to the standard VCCS, where these signals clearly differ from each other. For the VCCS in a two-channel version the first pulse modulation component in the modulator current is at a frequency of $4 / T_{c}$, instead of $2 / T_{c}$ and its magnitude is over 3-times lower compared to a one-channel converter.

A very adequate and reliable criterion of the quality of a converter output signal can be the converter control error given by the following equation:

$$
\varepsilon_{\mathrm{CTR}}=\sqrt{\frac{\overline{\left|u_{\mathrm{err}}\right|^{2}}}{\overline{\left|u_{\mathrm{ref}}\right|^{2}}}} 100 \%=\sqrt{\frac{\overline{\left|u_{\mathrm{ref}}-u_{\mathrm{fb}}\right|^{2}}}{\overline{\left|u_{\mathrm{ref}}\right|^{2}}}} 100 \% .
$$

Assuming nominal conditions of the simulation model work, the value is as follows: $\varepsilon_{\mathrm{CTR}}=5.88 \%: M=1, \varepsilon_{\mathrm{CTR}}=3.63 \%: M=2$. Thus, in sense of this criterion a two-channel 
VCCS makes it possible to improve the quality of the output current about $62 \%$ compared to a one-channel VCCS solution.

The simulation model has been "powered" by $3 \times 230 \mathrm{~V}, 50 \mathrm{~Hz}$ grid. The nominal DC output power of the rectifier system $P_{\mathrm{DC}, n}$ has been set at $6 \mathrm{~kW}$. While the current modulator is disabled the $T H D$ value for the power grid current $\left(i_{A}, i_{B}\right.$ and $\left.i_{C}\right)$ is approximately equal to $12.5 \%$ in a $5 \mathrm{kHz}$ band. This is the typical value for a standard 12-pulse rectifier. If the current modulator is enabled, the deformations of power grid currents (Fig. 7) are now of an over order less, i.e. $T H D=1.35 \%$ while the output power is equal to the nominal value and $T H D=2.93 \%$ while the output power is equal to the $10 \%$ of this one.

a)

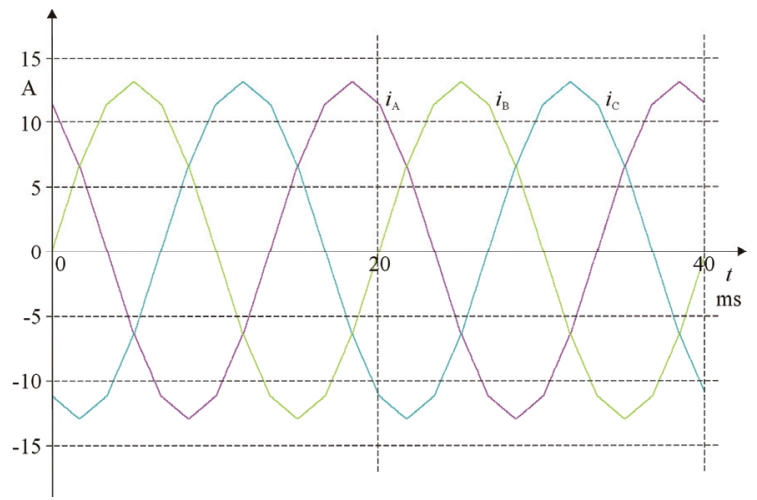

b)

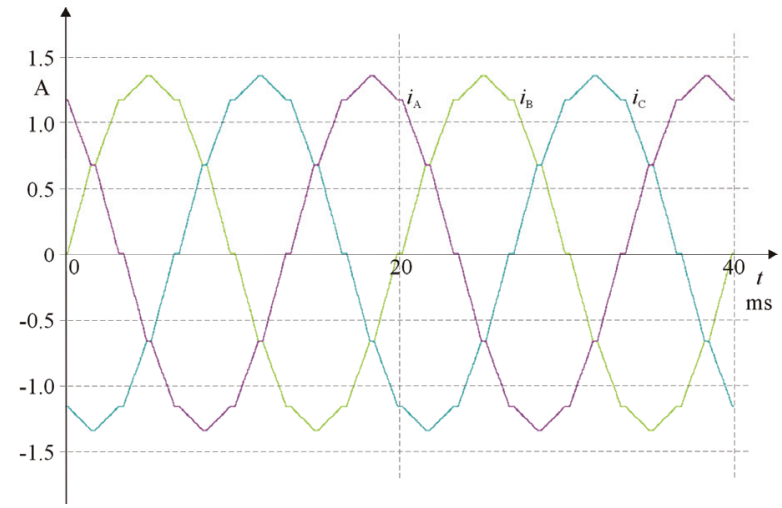

Fig. 7. Waveforms of power grid currents $i_{A}, i_{B}$ and $i_{C}$ in rectifier simulation model while current modulator is enabled and: a) output power is equal to the nominal value, b) output power is equal to $10 \%$ of nominal value

The impact of the number of converter channels on the quality of power grid currents can be evaluated on the basis of the $\varepsilon_{i}$ error. This one is related to the difference of a $i_{A}\left(i_{B}, i_{C}\right)$ current and its $1^{\text {st }}$ harmonics - similarly to the converter control error (8). A value of this error is a function of both, the rectifier output power $P_{\mathrm{DC}}$ and $M$ :

$$
\varepsilon_{i, M}=f\left(\frac{P_{\mathrm{DC}}}{P_{\mathrm{DC}, n}}\right): M=1,2 .
$$


The graph of this function is shown in Fig. 8. Also, the relationship of $\varepsilon_{i, 2}$ and $\varepsilon_{i, 1}$ is presented.

The quality of the power grid current in the case of utilization of the two-channel converter is increased about $10.8 \div 28.3 \%$ compared to the standard one, while its average value is equal to $11.8 \%$. In consequence, power loss in the rectifier system (mainly in transformers) can be reduced by means of a two-channel converter solution.

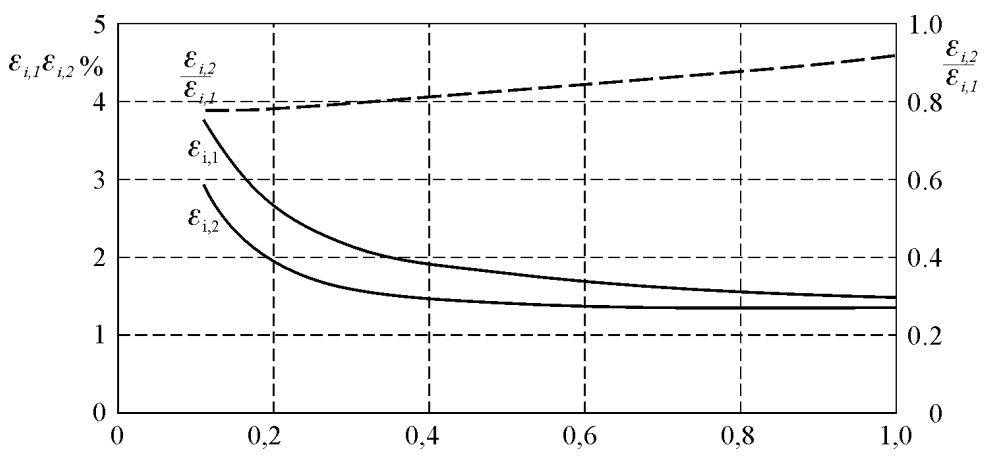

Fig. 8. Curves of $\varepsilon_{i}$ and reciprocal relationship of error functions $\left(\varepsilon_{i, 2} / \varepsilon_{i, 1}\right)$ while $M=1$ and $M=2$

\section{Laboratory model of current modulator}

The laboratory model of the part of a rectifier system has been investigated. The aim of the study has been the validation of theoretical assumptions and research results of a rectifier simulation model - mainly in the relation to the quality of the modulator current. The laboratory model has consisted of these blocks of the full rectifier circuitry which are on the grey background in Fig. 1. Its diagram has been consistent with Fig. 3. The secondary winding of the pulse transformer has been short circuited.

Basic technical parameters of the laboratory prototype are given below:

- DC link voltage in the multi-channel converter: $60 \mathrm{~V}$,

- nominal magnitude of the modulator current: $10 \mathrm{~A}$,

- inductance of the single coil in the VCCS: $1.15 \div 1.27 \mathrm{mH}$,

- signal sampling frequency in the control module: $20 \mathrm{kHz}$,

- PWM carrier frequency: $10 \mathrm{kHz}$.

The control module in the laboratory prototype has been based on the ALS-G3-1369 [18] DSP evaluation board with Analog Devices ADSP-21369 SHARC 8 DSP and two P3-5-550MFE LABINVERTERs [18] in the execution block.

Laboratory model tests have been carried out for the current modulator magnitude being in the range of $10 \% \div 100 \%$ of the nominal one. Also, two cases of converter configuration have been tested i.e. $M=1$ and $M=2$. In Fig. 9 and Fig. 10 exemplary waveforms in the laboratory prototype are shown while the current modulator magnitude is equal to the nominal one. Assuming nominal conditions of the laboratory model work, the value of the control error is as follows: 
$\varepsilon_{\mathrm{CTR}} \cong 9.8 \%$ while $M=1$ and $\varepsilon_{\mathrm{CTR}} \cong 5.9 \%$ while $M=2$. Thus, in sense of the control error criterion the two-channel VCCS makes it possible to improve the quality of the output current about $66 \%$ compared to a one-channel VCCS solution.

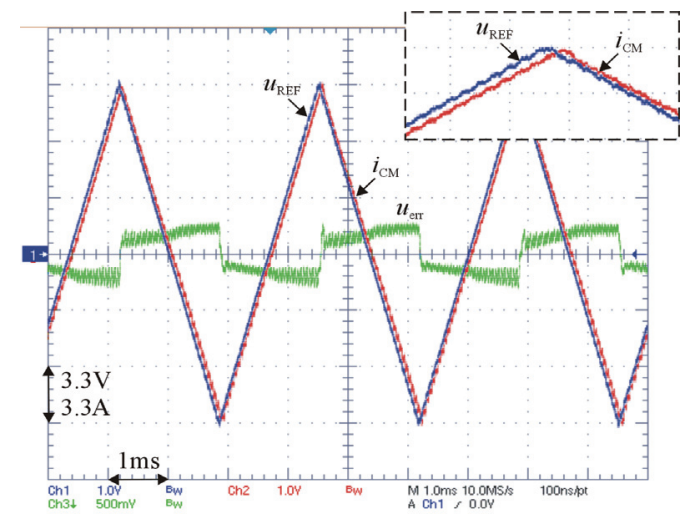

Fig. 9. Selected waveforms in laboratory prototype of current modulator: reference voltage, modulator current, and error signal while $M=1$

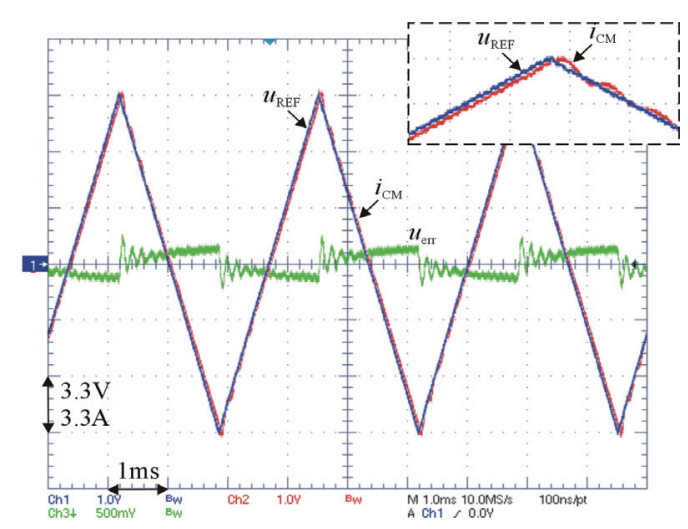

Fig. 10. Selected waveforms in laboratory prototype of current modulator: reference voltage, modulator current, and error signal while $M=2$; the current modulator magnitude is equal to the nominal one

\section{Conclusions}

In this study one of the ways leading to increasing the quality of power grid current of traditional diode rectifiers is presented. In order to obtain this a current modulator in the common DC circuit of two 6-pulses rectifiers has been utilized. The modulator is based on a wide-band power electronics controlled current source with a multi-channel converter. The control algorithm respects aliasing effects, having the influence on the system stability, taking place in sampled-data systems. They make it possible to maximize crucially effective gain of the regulator and, in consequence, better matching of the modulator output current in the reference 
signal. As a result the total $T H D$ factor of the power grid current is lowered approximately $11 \% \div 28 \%$, compared to the standard converter. It gives opportunity of decreasing power losses in the rectifier power grid transformers. The solution of a rectifier is especially attractive in the case of a higher power of the load, since power of the current modulator is equal to $2.5 \%$ of the rectifier DC output power. Thus, higher complexity of the proposed converter solution has only a minimal effect on the cost of the whole rectifier system.

\section{References}

[1] Siwczyński M., Jaraczewski M., Reactive compensator synthesis in time-domain, Bulletin of the Polish Academy of Sciences, Technical Sciences, vol. 60, no. 1, pp. 119-124 (2012).

[2] Gwóźdź M., Power Electronics Active Shunt Filter with Controlled Dynamics, Proc. of COMPEL: The International Journal for Computation and Mathematics in Electrical and Electronic Engineering, vol. 32, no. 4, pp. 1337-1344 (2013).

[3] Wilamowski B., Irwin J., Power Electronics and Motor Drives, CRC Press, (2011).

[4] Asiminoaei L., Aeloiza E., Enjeti P.N., Blaabjerg F., Shunt Active-Power-Filter Topology Based on Parallel Interleaved Inverters, IEEE Trans. on Industrial Electronics, vol. 55, no. 3, pp. 1175-1189 (2008).

[5] Demirdelen T., İnci M., Çağatay Bayindir K., Tümay M., Review of hybrid active power filter topologies and controllers, Power Engineering, Energy and Electrical Drives (POWERENG), Fourth International Conference, pp. 283-289 (2013).

[6] Jasiński M., Antoniewicz P., Kaźmierkowski M.P., Induction Motor Drive fed by PWM Rectifier/Inverter with Vector Control, Przegląd Elektrotechniczny (in Polish), no 6/2005, pp. 1-5 (2005).

[7] Lazaro A., Barrado A., Sanz M., Salas V., Olias E., New Power Factor Correction AC-DC Converter With Reduced Storage Capacitor Voltage, IEEE Trans. on Industrial Electronics, vol. 54, no 1, pp. 384-397 (2007).

[8] Agamy M., Jain P., A Three-Level Resonant Single-Stage Power Factor Correction Converter: Analysis, Design, and Implementation, IEEE Trans. on Industrial Electronics, vol. 56, no. 6, pp. 2095-2107 (2009).

[9] Mysiak P., Strzelecki R., A robust 18-pulse diode rectifier with coupled reactors, Bulletin of the Polish Academy of Sciences, Technical Sciences, vol. 59, no. 4, pp. 541-550 (2011).

[10] Krystkowiak M., Power rectifier system with improved rates with power electronics current modulator, Doctoral thesis (in Polish), Poznan University of Technology, Faculty of Electrical Engineering, Poznań (2009).

[11] Krystkowiak M., Gwóźdź M., Simulation and Experimental Models of 3-Phase Diode Rectifier with Current Modulation in DC Circuit, Przegląd Elektrotechniczny, ISSN 0033-2097, vol. 1, pp. 75-79 (2011).

[12] Gwóźdź M., Stability of Discrete Time Systems on Base Generalized Sampling Expansion, Quarterly Elektryka, Politechnika Śląska, vol. 1 (217), Gliwice, pp. 29-40 (2011).

[13] Huth S., DC/DC converters in Parallel Operation with digital load distribution control, Proceedings of the IEEE International Symposium on Industrial Electronics, vol. 2, pp. 808-813 (1996).

[14] Papoulis A., Generalized Sampling Expansion, Proceedings of the IEEE Transactions on Circuits and Systems, vol. 24, issue 11, pp. 652-654 (1977).

[15] Mirkin L., Palmor Z.J., Control Issues in Systems with Loop Delays, The Handbook of Networked and Embedded Control Systems (D. Hristu-Varsakelis and W.S. Levine, eds.), Birkhäuser, pp. 627-648 (2005).

[16] Meinsma G., Mirkin L., Sampling from a System-Theoretic Viewpoint: Part I - Concepts and Tools, Part II-Noncausal Solutions, IEEE Trans. on Signal Processing, vol. 58, no.7, pp. 3578-3606 (2010).

[17] Kaczorek T., Control and systems theory, Wyd. Naukowe PWN (in Polish), W-wa (1999).

[18] Product page of ALFINE-TIM: http://analog.alfine.pl, accessed April 2014. 